\title{
Results on Circular-Arc Bigraphs
}

(Extended Abstract)

\author{
Fabricio Schiavon Kolberg ${ }^{1 *}$, Marina Groshaus ${ }^{2 \dagger}$ \\ André Luiz Pires Guedes ${ }^{1}$, Renato Carmo ${ }^{1}$ \\ ${ }^{1}$ Universidade Federal do Paraná, Curitiba, Brazil \\ ${ }^{2}$ Universidad de Buenos Aires / CONICET, Buenos Aires, Argentina.
}

\begin{abstract}
We present a series of results related to the structural properties of the bipartite graph class known as circular-arc bigraphs. We also propose the definition of a Helly circular-arc bigraph subclass, based on a concept known as bipartite-Helly, along with a few results related to its structural properties.
\end{abstract}

\section{Introduction}

A circular-arc graph is a graph that admits an intersection model of arcs on a circle. Arising as a generalization of interval graphs, the class has been extensively studied by many researchers since 1964 [Lin and Szwarcfiter 2009], yielding a plethora of relevant results. Trotter and Moore [Trotter and Moore 1976] presented infinite sets of minimally non circular-arc graphs. Francis et al. [Francis et al. 2014] presented a characterization of circular-arc graphs, along with a certifying recognition algorithm. Furthermore, Hell and Huang [Hell and Huang 2004] characterized two-clique circular arc graphs as the complements of interval bigraphs. Soulignac, in his thesis [Soulignac 2010], characterized different circular-arc subclasses, including the Helly subclass, based on the concept of Helly families [Helly 1923]. A linear-time algorithm for recognition of the class was presented by McConell [McConnell 2003], based on the so-called circular-arc matrices.

The concept of circular-arc bigraphs arises as a bipartite variation of the circulararc concept, similar to interval bigraphs with relation to interval graphs. We treat circulararc bigraph representations as bi-circular-arc models $\mathcal{B}=(C, \mathbb{I}, \mathbb{E})$, where $C$ is a circle, and $\mathbb{I}, \mathbb{E}$ are families of arcs over $C$. It is then said that $\mathcal{B}$ represents a bipartite graph $G=(U, V, E)$ if there is a one-to-one correspondence between $U$ and $\mathbb{I}$, and one between $V$ and $\mathbb{E}$, such that, for every $u \in U$ and every $v \in V,\{u, v\} \in E$ if and only if the arc corresponding to $u$ in $\mathbb{I}$ and the one corresponding $v$ in $\mathbb{E}$ intersect.

Being a rather recent subject, it has yet to be as extensively studied as the other classes cited. Basu et al. [Basu et al. 2013] made a series of characterizations of circulararc bigraphs, as well as the proper and unit subclasses, based on the so-called biadjacency matrices. Das et al. [Das and Chakraborty 2015] found a forbidden structure characterization of proper circular-arc bigraphs and interval bigraphs.

In this paper, we present a handful of results about the structural properties of circular-arc bigraphs, as well as the Helly subclass, which we introduce. Our study's main focus were the structural properties of circular-arc bigraphs, based on their graph structures instead of matrix structures. A handful of potentially useful results were found, which we present in the next section.

\footnotetext{
*Partially supported by Coordenação de Aperfeiçoamento Pessoal de Nível Superior, CAPES

${ }^{\dagger}$ Partially supported by ANPCyT PICT-2013-2205 and CONICET
} 


\section{Results on Circular-Arc Bigraphs}

To represent circular arcs on a circle $C$ of length $n$, we represent each point as a real number $0 \leq r<n$, which denotes a clockwise offset through the circle from a fixed zero point in $C$. It is then possible to denote a circular arc by a pair $\left(r_{1}, r_{2}\right)$ of real numbers, where the arc is traced clockwise from point $r_{1}$ to point $r_{2}$, respectively called the counter-clockwise and clockwise endpoints of the arc. Furthermore, in the enunciation of the following results, every index sum is circular, which means that in an enumerated set $\left\{a_{1}, \ldots, a_{n}\right\}$, we have $a_{n+1}=a_{1}$ and $a_{1-1}=a_{n}$.

Lemma 1. Let $B=(U, V, E)$ be a bipartite graph, with $|V|=n$ and $|U|=m$. If there is an ordering $S=\left(v_{1}, \ldots, v_{n}\right)$ of $V$ such that, for every $u \in U$, the neighborhood of $u$ is circularly consecutive with relation to $S$, then $B$ is a circular-arc bigraph.

Proof. Start with a circle $C$ of length $n+1$. For each $v_{i} \in V$, draw an $\operatorname{arc}(i, i+1) \in \mathbb{I}$ to represent it. Then, for each $u \in U$ whose neighborhood ranges from $v_{i}$ to $v_{j}$, we draw an $\operatorname{arc}\left(i+\frac{1}{2}, j+\frac{1}{2}\right) \in \mathbb{E}$ to represent it. $(C, \mathbb{I}, \mathbb{E})$ corresponds to $B$.

Lemma 1 implies that graphs such as generalized crowns and bipartite permutation graphs are all circular-arc bigraphs.

Definition 1. Let $a>0$ and $0 \leq b<a$. The generalized crown graph $S_{b}^{a}$ is a bipartite graph with vertex set $\left\{u_{0}, \ldots, u_{a-1}\right\} \cup\left\{v_{0}, \ldots, v_{a-1}\right\}$, such that the neighborhood of $v_{i}$ is $U-\left\{u_{i}, \ldots, u_{i+b}\right\}$.

Definition 2. Let $G=(V, E)$ be a graph. Then $G$ is a permutation graph if there exist two permutations $P_{1}, P_{2}$ of its vertex set such that, if the index of $v$ in $P_{1}$ is less than that of $w$, then $\{v, w\} \in E$ if and only if the index of $v$ in $P_{2}$ is greater than that of $w . A$ bipartite permutation graph is simply a permutation graph that is bipartite.

Let $\mathbb{A}$ be a family of arcs on a circle $C$. An $\operatorname{arc} B$ of $C$ is said to minimally intersect $A \in \mathbb{A}$ if it intersects $A$ without intersecting another arc $A^{\prime} \in \mathbb{A}$ such that $A^{\prime} \subset A$. Lemma 2 allows us to describe a type of forbidden structure for the class.

Lemma 2. Let $0<m<n$, and let $\mathbb{A}$ be a family of arcs on a circle $C$, with $|\mathbb{A}|=n$, such that no two arcs have coinciding endpoints. There are at most $n$ subfamilies $\mathbb{A}^{\prime} \subset \mathbb{A}$ such that $\left|\mathbb{A}^{\prime}\right|=m$ for which it is possible to draw an arc on $C$ that intersects every arc of $\mathbb{A}^{\prime}$ without intersecting any of $\mathbb{A}-\mathbb{A}^{\prime}$.

Proof. Let $B$ be an arc on $C(B \notin \mathbb{A})$ that intersects exactly $m$ arcs of $\mathbb{A}$. Let $\mathbb{A}^{\prime}$ be the family of arcs intersected by $B$, and let $S=\left(A_{1}, \ldots, A_{m}\right)$ be the order in which the counter-clockwise endpoint of each arc in $\mathbb{A}^{\prime}$ is first encountered by trailing the circle clockwise from the clockwise endpoint of $B$. Let $A_{i}$ be the first arc in the order $S$ that is minimally intersected by $B$. We shall call $A_{i}$ the first minimally intersected arc of $B$. Notice that $\mathbb{A}^{\prime}$ contains all arcs that contain $A_{i}$. Let $k>0$ be the number of such arcs. In particular, notice that every arc in $\left\{A_{1}, \ldots, A_{i-1}\right\}$ contains $A_{i}$, otherwise, either they would be minimally intesercted themselves, or they'd contain another arc that is minimally intersected and comes before $A_{i}$ in $S$. The other arcs that $\mathbb{A}^{\prime}$ contains will be exactly the first $m-k$ arcs that are not contained in (nor contain) $A_{i}$ and whose counter-clockwise endpoints come immediately after the counter-clockwise endpoint of $A_{i}$. Therefore, if $A_{i}$ is the first minimally intersected arc of a new $\operatorname{arc} B$ that intersects $m$ arcs, $\mathbb{A}^{\prime}$ will always be the same. That implies that for each arc $A \in \mathbb{A}$, there is at 
most one subfamily of $m$ arcs which can be intersected by a new arc with $A$ being the first minimally intersected arc (there might also be none, for instance, if an arc is contained in more than $m-1$ arcs). Therefore, there are at most $n$ subfamilies of $m$ arcs that can be exclusively intersected by a new arc.

Corollary 3. Let $B=(U, V, E)$ be a bipartite graph, such that $|U|=n,|V|=n+1$, and there is a $k$ such that $|N(v)|=k$ for all $v \in V$. If the neighborhoods of the vertices of $V$ are all pairwise distinct, then $B$ is not a circular-arc bigraph.

Figure 1 contains a handful of forbidden subgraphs we found for the class.
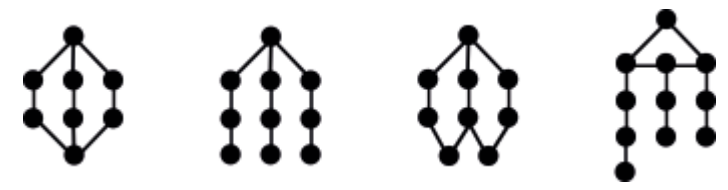

Figure 1. List of forbidden subgraphs we found for the circular-arc bigraph class.

\subsection{Studies on the Helly Subclass}

The concept of Helly families [Helly 1923] lends itself to the definition of many extensively studied graph classes [Szwarcfiter 1997, Groshaus and Szwarcfiter 2007, Groshaus and Szwarcfiter 2008]. An adaptation of the definition of Helly families, known as bipartite-Helly, was formulated by Groshaus and Szwarcfiter [Groshaus and Szwarcfiter 2010], and allows us to define Helly-like properties between pairs of families. For the results in this section, we first introduce interval bigraphs in Definition 3, and then Helly circular-arc bigraphs and Helly interval bigraphs in Definition 4, loosely based on the bipartite-Helly concept.

Definition 3. A bipartite graph $B=(U, V, E)$ is an interval bigraph if it admits a biinterval representation. A bi-interval representation of $B$ is a mapping between $U \cup V$ and a family of intervals on the real number line, such that $u \in U$ and $v \in V$ are neighbors if and only if their corresponding intervals intersect.

For the following definition, we consider a biclique of a given graph to be a maximal bipartite-complete induced subgraph.

Definition 4. A bipartite graph $B$ is a Helly circular-arc bigraph if it admits a Helly bicircular-arc model. A bi-circular-arc model $(C, \mathbb{I}, \mathbb{E})$ is Helly if, for any biclique in the graph it represents, there is a point $p \in C$ such that all arcs corresponding to vertices of the biclique contain $p$. A bipartite graph $B$ is a Helly interval bigraph if it admits a Helly bi-interval representation. A bi-interval representation is Helly if, for any biclique of the graph it represents, the intervals corresponding to the vertices of the biclique all contain a common point $p$ in the real number line.

It is easy to verify that both classes are hereditary over induced subgraphs, and that Helly interval bigraphs are a proper subclass of Helly circular-arc bigraphs. Lemma 4 presents a sufficient condition for a graph to be a Helly circular-arc bigraph. We denote by $\left(C_{2 k}, S_{\ell}\right)$ the graph obtained by adding $\ell$ isolated vertices to a $C_{2 k}$.

Lemma 4. Let $G$ be a bipartite graph. If $G$ is $K_{1,3}$-free and $\left(C_{2 k}, S_{\ell}\right)$-free (with $k>2$ ), then $G$ is a Helly circular-arc bigraph. 
Proof. If the graph contains a $C_{2 n}$, for $n>2$, and doesn't contain any induced $K_{1,3}$ nor any isolated vertices, then its only component is the $C_{2 n}$, which can easily be verified to be a Helly circular-arc bigraph. Now suppose the graph does not contain a $C_{2 n}$, for $n>2$. Then, since the graph is $K_{1,3}$-free, every single one of its components is either a path or a $K_{2,2}$. It is easy to verify that both paths and the $K_{2,2}$ are Helly interval bigraphs, meaning that a graph that has those graphs as connected components also is.

Lemma 4 is a simple sufficient condition for the Helly subclass, serving to prune the search space for forbidden structures. It is known to not be necessary, since $K_{1,3}$ by itself is a Helly circular-arc bigraph.

\section{References}

Basu, A., Das, S., Ghosh, S., and Sen, M. (2013). Circular-arc bigraphs and its subclasses. Journal of Graph Theory, 73(4):361-376.

Das, A. K. and Chakraborty, R. (2015). Algorithms and Discrete Applied Mathematics: First International Conference, CALDAM 2015, Kanpur, India, February 8-10, 2015. Proceedings, chapter New Characterizations of Proper Interval Bigraphs and Proper Circular Arc Bigraphs, pages 117-125. Springer International Publishing, Cham.

Francis, M. C., Hell, P., and Stacho, J. (2014). Forbidden structure characterization of circular-arc graphs and a certifying recognition algorithm. CoRR, abs/1408.2639.

Groshaus, M. and Szwarcfiter, J. (2007). Biclique-helly graphs. Graphs and Combinatorics, 23:633 - 645 .

Groshaus, M. and Szwarcfiter, J. (2008). On hereditary helly classes of graphs. Discrete Mathematics \& Theoretical Computer Science, 10(1).

Groshaus, M. and Szwarcfiter, J. L. (2010). Biclique graphs and biclique matrices. Journal of Graph Theory, 63(1):1-16.

Hell, P. and Huang, J. (2004). Interval bigraphs and circular arc graphs. J. Graph Theory, 46:313-327.

Helly, E. (1923). Über Mengen konvexer Körper mit gemeinschaftlichen Punkten. Jahresber. Dtsch. Math.-Ver., 32:175-176.

Lin, M. C. and Szwarcfiter, J. L. (2009). Characterizations and recognition of circular-arc graphs and subclasses: A survey. Discrete Mathematics, 309(18):5618-5635. Combinatorics 2006, A Meeting in Celebration of Pavol Hell's 60th Birthday (May 1-5, 2006).

McConnell, R. M. (2003). Linear-time recognition of circular-arc graphs. Algorithmica, 37(2):93-147.

Soulignac, F. J. (2010). Sobre Grafos Arco-Circulares Propios y Helly. PhD thesis, Universidad de Buenos Aires, Facultad de Ciencias Exactas y Naturales, Departamento de Computacion.

Szwarcfiter, J. L. (1997). Recognizing clique-Helly graphs. Ars Comb., 45:29-32.

Trotter, Jr., W. T. and Moore, Jr., J. I. (1976). Characterization problems for graphs, partially ordered sets, lattices, and families of sets. Discrete Mathematics, 16(4):361381. 